7th International Workshop on Astronomy and

Relativistic Astrophysics (IWARA 2016)

International Journal of Modern Physics: Conference Series

Vol. 45 (2017) 1760039 (5 pages)

(C) The Author(s)

DOI: $10.1142 / S 2010194517600394$

\title{
Quark-hadron Phase Transition in Proto-Neutron Stars Cores Based on a Non-local NJL Model
}

\author{
German Malfatti, Milva Orsaria \\ Consejo Nacional de Investigaciones Científicas y Técnicas (CONICET) \\ Godoy Cruz 2290, 1425 Buenos Aires, Argentina \\ Facultad de Ciencias Astronómicas y Geofísicas \\ Universidad Nacional de La Plata (UNLP) \\ Paseo del Bosque S/N (1900), La Plata, Argentina \\ germanmalfatti@gmail.com, morsaria@fcaglp.unlp.edu.ar \\ Gustavo A. Contrera \\ Consejo Nacional de Investigaciones Científicas y Técnicas (CONICET) \\ Godoy Cruz 2290, 1425 Buenos Aires, Argentina \\ Facultad de Ciencias Astronómicas y Geofísicas \\ Universidad Nacional de La Plata (UNLP) \\ Paseo del Bosque S/N (1900), La Plata, Argentina \\ Facultad de Ciencias Exactas \\ Universidad Nacional de La Plata (UNLP) \\ calle 49 y 115, La Plata, Argentina \\ contrera@fisica.unlp.edu.ar \\ Fridolin Weber \\ Department of Physics \\ San Diego State University (SDU) \\ 5500 Campanile Drive, 92182 San Diego, CA, USA \\ Center for Astrophysics and Space Sciences \\ University of California \\ 92093 San Diego, La Jolla, CA, USA \\ fweber@mail.sdsu.edu
}

Published 15 August 2017

\begin{abstract}
We study the QCD phase diagram using a non-local SU(3) NJL model with vector interactions among quarks. We analyze several thermodynamic quantities such as entropy and specific heat, and study the influence of vector interactions on the thermodynamic properties of quark matter. Upon imposing electric charge neutrality and baryon number conservation on the field equations, we compute models for the equation of state of the
\end{abstract}

This is an Open Access article published by World Scientific Publishing Company. It is distributed under the terms of the Creative Commons Attribution 4.0 (CC-BY) License. Further distribution of this work is permitted, provided the original work is properly cited. 


\title{
G. Malfatti et al.
}

\begin{abstract}
inner cores of proto-neutron stars by providing a non-local treatment of quark matter for astrophysics.
\end{abstract}

Keywords: Phase Diagram, Equation of State, Neutron Stars.

PACS numbers: 26.60.+c, 26.60.-c, 05.70

\section{Introduction}

It is known that quantum chromodynamics (QCD), has two very important properties, namely asymptotic freedom and confinement. The former implies that at high momentum transfers, the quarks behave as quasi free particles, i.e., the interaction between two quarks due to gluon interchange can be treated using perturbation theory. For this momentum range, the dispersion processes can be then be calculated with a very good precision. By contrast, at low momentum exchange among the quarks ( $\lesssim 1 \mathrm{GeV}$ ) QCD is highly nonlinear and leads to quark confinement. Several approximate methods have been developed to study the physical processes among quarks in the low momentum range of QCD. Among them is lattice QCD, which tries to solve the QCD equations of motion numerically on a discretized spacetime grid ${ }^{1}$. This method, however, presents problems if extended to finite chemical potentials ${ }^{2}$. Another option is to use effective QCD models, like the Nambu-JonaLasinio model (NJL), which is described by a Lagrangian which accounts for the main features of QCD at low energies. The advantage of NJL models is that they can be extended to finite chemical potentials easily. Local as well as non-local extensions of the model have been studied in the literature (see, for instance, Ref. ${ }^{3}$, and references therein).

Non-local extensions of the NJL model at zero temperature have been used to study hybrid stars with and/or without a quark-hadron mixed phases in their inner cores (see Refs. ${ }^{3-5}$, and references therein). Here, we present an extension of such studies to finite temperatures in order to explore the role of a quark-hadron phase transition for proto-neutron stars or core collapse supernovae.

\section{2. n3PNJL Model and Phase Diagram}

The Euclidean effective action of the model presented in this paper is for the nonlocal 3-flavor Polyakov NJL model, including the vector interactions among quarks. This action is given by

$$
\begin{gathered}
S_{E}=\int d^{4} x\left\{\bar{\psi}(x)\left[-i \gamma_{\mu} D_{\mu}+\hat{m}\right] \psi(x)-\frac{G_{s}}{2}\left[j_{a}^{S}(x) j_{a}^{S}(x)+j_{a}^{P}(x) j_{a}^{P}(x)\right]\right. \\
-\frac{H}{4} T_{a b c}\left[j_{a}^{S}(x) j_{b}^{S}(x) j_{c}^{S}(x)-3 j_{a}^{S}(x) j_{b}^{P}(x) j_{c}^{P}(x)\right] \\
\left.-\frac{G_{v}}{2}\left[j_{v}^{\mu}(x) j_{v}^{\mu}(x)\right]+\mathcal{U}[\mathcal{A}(x)]\right\},
\end{gathered}
$$

where $j_{a}^{S}(x), j_{a}^{P}(x)$ and $j_{v}^{\mu}(x)$ are the scalar, pseudoscalar and vector currents, respectively, and $\mathcal{U}[\mathcal{A}(x)]$ is the effective potential related with the Polyakov loop. 
To extend the model to finite temperatures we use the Matsubara imaginary time formalism ${ }^{6}$. After the bosonization of Eq. (1) we obtain the grand canonical potential for the mean field approximation, from which the phase diagram (Fig. (1)) and thermodynamic quantities such as the specific heat, $C_{v}$, and specific entropy, $s$, (Fig. (2)) can be calculated.
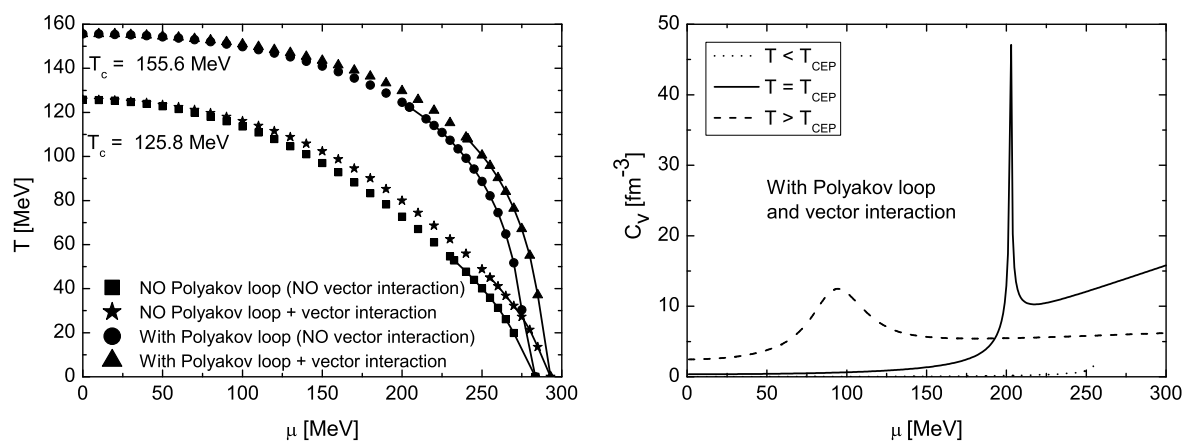

Fig. 1. Phase diagram of QCD matter. Left panel: temperature, $T$, as a function of chemical potential, $\mu$. Right panel: specific heat, $C_{v}$, as a function of $\mu$. (CEP stand for critical end point.) The impact of the Polyakov loop and vector interactions on $C_{v}(\mu)$ is very small and therefore not shown separately. The locations of irst-order phase transitions are shown by solid lines.

The quark current masses and coupling constants in Eq. (1) can be chosen so as to reproduce the phenomenological values of the pion decay constant, $f_{\pi}$, and the meson masses $m_{\pi}, m_{\eta}$, and $m_{\eta^{\prime}}$, as described in Ref. ${ }^{7}$. In this work we choose the strange quark mass to the updated value of $m_{s}=95 \mathrm{MeV}$, with the values of the other parameters given by $\Lambda=1071.38 \mathrm{MeV}, G_{s} \Lambda^{2}=10.78, H \Lambda^{5}=-353.29$ and $m_{u}=m_{d}=3.63 \mathrm{MeV}$. The vector interaction coupling constant, $G_{v}$, has been
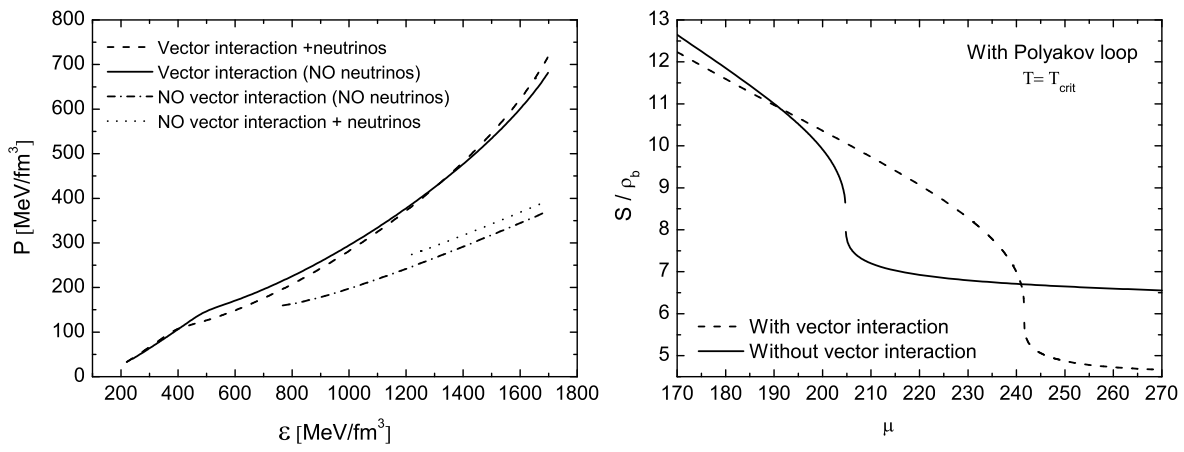

Fig. 2. Models for the equation of state (pressure versus energy density) of proto-neutron star matter (left panel), and entropy per baryon (in units of the Boltzmann constant) versus chemical potential (right panel). 


\section{G. Malfatti et al.}

assigned a canonical value of $G_{v}=0.5 G_{s}$. Finally, we mention that the parameter $\Lambda$ determines the range of the non-locality in momentum space.

Because of their short mean-free paths, the neutrinos produced in the core of a proto-neutron star are prevented from leaving the star on a dynamical time scale. The number of lepton-to-baryon ratio of such matter is around $Y_{L_{e}} \simeq 0.4$, but the exact value depends on the efficiency of electron capture reactions during the gravitational collapse of the supernova. The number of muons per baryon is $Y_{L_{\mu}}=0$, because no muons are present in the stellar matter prior to the trapping. On a timescale of 10 to 20 seconds, the neutrinos diffuse from the star, but leave behind much of their energy which causes significant heating of the ambient matter ${ }^{8}$. The temperatures generally achieved in the inner $50 \%$ of the stellar core at the peak of the heating are in the range of 30 to $50 \mathrm{MeV}$. Following the heating, the star cools by radiating neutrino-anti-neutrino pairs, and consequently the temperature drops off to $\sim 1 \mathrm{MeV}$ within minutes. A model for the equation of state of such matter, which describes the inner cores of proto-neutron stars, is shown in Fig.(2).

\section{Conclusions}

We have constructed models for the equation of state of hot quark matter in the framework of the n3PNJL model. Knowledge of the equation of state, a possible critical end point (CEP), and the nature of the phase transition in the QCD phase diagram itself are critical for a better understanding of the strongly interacting matter at high densities and/or temperatures. We have found that quark confinement as described by the Polyakov loop modifies the phase diagram by increasing the transitions temperatures. The inclusion of vector interactions among quarks, on other hand, changes the location of the CEP. Also, vector interactions stiffen the equation of state, while the inclusion of neutrinos has the opposite effect (softens the equation of state).

One of the main motivations of this study concerns the possible occurrence of quark matter inside of proto-neutron stars. One of the mechanism for the formation of quark matter in the inner cores of such stars could be the nucleation of quark matter drops due to the high pressure environment (high compression) that exists in the centers of proto-neutron star ${ }^{9}$. Such an investigation, as well as the effect of neutrino trapping in hot beta-stable quark-hybrid matter is currently begin carried out.

\section{Acknowledgments}

G. M., G. A. C. and M.O. thank CONICET and UNLP for financial support. M. O. acknowledges financial support from American Physical Society's International Research Travel Award Program. F. W. is supported by the National Science Foundation (USA) under Grant PHY-1411708. 


\section{References}

1. F. J. Ynduráin, The theory of quarks and gluon interactions (Springer-Verlag, Heilderberg, 1999).

2. F. Karsch, Springer Lect. Notes Phys. 583, 209 (2002).

3. I. F. Ranea-Sandoval, S. Han, M. G. Orsaria, G. G. Contrera, F. Weber, and M. G. Alford, Phys. Rev. C 93, 045812 (2016).

4. W. M. Spinella, F. Weber, G. A. Contrera, and M. G. Orsaria, it EPJA 52, 61 (2016).

5. S. M. de Carvalho, R. Negreiros, M. Orsaria, G. A. Contrera, F. Weber, and W. Spinella, Phys. Rev. C 92, 035810 (2015).

6. J. I. Kapusta and C. Gale, Finite-Temperature Field Theory: Principles and Applications (Cambridge Monographs on Mathematical Physics, Cambridge: Cambridge University Press, 2006).

7. G. A. Contrera, D. Gomez Dumm, and N. N. Scoccola, Phys. Rev. D 81, 054005 (2010).

8. T. J. Burrows, A. Mazurek, and J. M. Lattimer. Astrophys. J. 251, 325 (1981); A. Burrows and J. M. Lattimer, Astrophys. J. 307, 178 (1986).

9. G. Lugones, Eur. Phys. J. A 52, 53 (2016). 